\title{
Issues of Ethics in Developing World
}

\author{
Marriam Gul Thaheem ${ }^{1 *}$ and Mustafa Aslam² \\ ${ }^{1}$ Clinical Oncologist, Jinnah post graduate medical center, Pakistan \\ ${ }^{2}$ Forensic physician and Ethicist, The Aga Khan University, Pakistan
}

Submission: June 26, 2017; Published: August 03, 2017

*Corresponding author: SMarriam Gul Thaheem, Clinical Oncologist, Ethicist, Jinnah post graduate medical center, Karachi, Pakistan, Email: marrygul@hotmail.com

\section{Introduction}

Ethical reports require conducting a clinical research aimed to minimize the possibility of exploitation of human subjects. The role of ethical requirement of clinical research to ensure the possibility of human subjects for them not is being used as the mere means while they contribute to the social good. Various guidelines have been designed and implemented in order conduct clinical trials and research in order to particularly be ethical and to avoid any immoral or injustice to the subjects involved in the trial that is research participants, Nuremberg code Belmont report, declaration of Helsinki and international ethical guidelines for bio medical research involving human subjects are few of those which were considered as standard of check and balance before the active implementation and practice of the clinical research. Nuremberg code, is mainly concerned to the judicial decision making part and also focused on favorable risk benefit ratio and also concerned with need for consent but has not shown any concern on subject selection or independent review.

\section{Opinion}

The declaration of Helsinki, this mainly showed its concerns towards the physician's behavior and the conduct of the research with patients and also has its own concerns regarding favorable risk benefit ratio and independent review. It also has emphasis on distinction between therapeutic and non therapeutic research which is either ejected or has remained UN noticed by other documents. The Belmont report, is designed in regard to generate specific rules and regulations in response to highlighted research scandals. Also focused on vulnerable researches not targeted for risky researchers, also involves informed consent and favorable risk benefit ratio. CIMOS council for international organizations of medical sciences has designated or assigned the rules of declaration of Helsinki's to be implemented and followed in developing countries in particular for the trials of drugs and vaccines.
Globally as well as in developed countries there are mainly seven parameters or ethical requirements are considered as standard and are designed a sketch a frame work for determining whether the clinical research is ethical or not? These severe parameters or hallmarks are checked from the conception to the formulation and implementation of the research project. They are designed to guide the ethical development, implementation and review of the individual clinical protocols and also help to elucidate the clinical standards. All these requirements are meant to be universal, although a little amendments in adaptations to difficult cultures, economical settings, health conditions and requirements and other cultural values. The proper and prior consideration of these seven points which are ideally required to conceptualize prior to the start of the trial thus absorbing most of the forth coming issues foresee and dealt pre trial and have great importance globally and while planning a clinical trial in any developing country as well. Value in order to make a clinical research valuable and ethically sound means the design of a clinical research shall be capable to evaluate a diagnostic and therapeutic intervention or would eventually lead to improve the health standard and general well being. The scientific value of a clinical research design includes clinical research with non generalizable results, authentic proven results. In order to validate the scientific value of the clinical research only if the society will gain knowledge which requires sharing of the results and outcome of the clinical research whether positive or negative, only then exposing of human subjects to the clinical research be justified. Sharing of results shall be ensured prior. It is therefore important to enforce scientific and social value be an ethical requirement in order to avoid exploitation and to ensure the responsible use of finite resource. A comparative evaluation of the funding priorities and allocation of funds may be necessary. Scientific Validity, that is to deal with the use of accepted and proven scientific methods and principals, including statistical techniques in order to valid and reliable data/outcomes. According to CIOM guidelines if 
scientifically unsound researches conducted on human subjects, it is therefore highly unethical as it carries high risk of exposing human subjects or maybe inconvenience to no purpose. Clinical research protocol must have clear scientific objectives following the acceptable principals, methods, sufficient power to definitely test the objective. Scientific validity prime importance unless clinicians and researchers are not sure whether the new treatment is better while believe it is. Scientific validity ensures the scientific designs which realize the scientific goals and objectives by guaranteeing the research candidates for all the health care interventions for which they are entitled being a part of the research. Scientific design shall also validate the scientific design of research that realizes the social values of the primary beneficiaries of the research. The research study should cater all the feasibilities within the social, political and cultural context that makes reasonably sustainable improvements in the local health care and physical infrastructure for the provision of an affordable drug. Fair subject selection, it is considered as a great challenge et o make a fair selection of the subject by targeting tribes, villages and neighboring cities specially in the developing countries, scientific reasoning of high prevalence in order to chose a particular group of people or community in order to identify commonly spread infections, particular combination of diseases, living standards of particular community, rates of transmissions of infections. Just being dependant on scientific considerations only will not be sufficient enough to target a community or the individuals which are to be selected for the participation of the study, therefore it is always recommended to focus or select an already established community or if not so at least it shall be a community that should be capable enough to establish a system for identifying the legitimate representatives and shall be made responsible to share the responsibility of planning in conducting and implementation of the health research through health system improvements. It is usually seen and historically observed that populations which are poor or powerless to defend their own rights are usually targeted for the research purpose therefore social marginalization, political powerlessness and economic deprivation must be taken as a basic consideration as to determine the vulnerability of the community/society. Risk-benefit ratio favorable. It is one of the prime ethical issues that all the clinical research methodologies at the time being or rather at the time of policy making shall outweigh the riskbenefit ratio. Although assessment of favorable risk-benefit ratio is one of the prime concern both ethically and scientifically but still two unique bench marks of favorable risk-benefit ratio needs to be taken under consideration when it comes to developing countries. Ist context in which they live; It must be favorable for the individuals to outweigh the risk-benefit ratio in context of their living habits and circumstances, therefore there is a direct effect on the underlying risks of that potential disease, it may vary in incidence, drug resistance, genetic susceptibility and other social and environmental factors. This ratio may be highly vulnerable for example; when participants confront higher risks of disease, greater potential benefits may justify greater risks in the research design and policy., on the other hand the ratio of risk and benefit may justify greater risk in the research design and policy, on the other hand the ratio of risk and benefit may highly go in favor of the community where the social value is in favor of research though but may be unfavorable when political values are low. 2nd The risk-benefit ratio shall also go in favor of community to make certain assessments such as antibiotic resistance. In such case the community shall be capable to determine whether the risks are acceptable in comparison to the benefit which are to be expected during and after the course of conduct of the clinical research trial and thus the results obtained. Independent Review, Independent ethical review of all the clinical research protocol is necessary and this is in regard to cater the researcher's methodology, policy and conflict of interest and to ensure the public/community accountability as well. There are certain scenarios in which in addition to IRB, institutional review board and ERC, ethical review committee certain other regulatory approvals may also be necessary. Review must be impartial, independent and competent. At several point and time the conflict may arise in terms of having different guidelines or different requirements of regulatory authorities which may be insensitive to particular social and cultural circumstances in the developing countries, so can be resolved by involving the other regulatory local systems in order to settle the issue as there is hardly any fundamental issue or disagreement which cannot be resolved, rather they are widely accepted. Informed Consent, it is been considered as a principal of ethical clinical research over a century. There are five bench marks of evaluating an informed consent. Local community shall establish a system to identify, establish and implement the incentives and recruitments, procedures for the participating community in consistence with the social cultural and political practices.

1. Dissemination of the information (correct) is highly sensitive and shall be done in local language and appropriate analogies shall be considered in order to make it simple and understandable to the participants.

2. Leaders of the villagers or leaders of extended family or heads of families may be required to be briefed about the nature of the research before researchers could invite the participants.

3. Consent taking procedure is important in local community to use the individual's autonomy to voluntarily participate and withdraw or refuse the participation to the research. Respect of participant and the Research community.

Once the clinical research policy and project is ready to be implemented practically the researchers are held responsible for an ongoing obligation towards the study participants, former participants, and the host community as well. An initial obligation on behalf of research is to develop and implement the procedure in order to maintain the confidentiality of all the information of the research participant that is been collected throughout the course of research. It is considered essential 
responsibility and obligation of the researcher, or if there is little variation in certain cases like HIV research or some other researches it is highly obligatory to inform the participant that there is no $100 \%$ guarantee about the confidentiality. Respect of the participant's decision to withdraw from the study shall be taken care of and community and the participants shall also be informed about new information and new discoveries about the risks and benefits of the research.

In order to maintain the privacy and confidentiality of the data different measures are to be taken in order to avoid the deliberate breach of confidentiality and the first step to avoid this is to inform study participants, the authorities or the researchers. The precautions to be taken depend upon the nature of the project and also to the potential benefit's from the information to the study participants or the community, therefore there are several levels of identification and accountability of the information by giving a code to the data files and to remove any sort of links between the code and the personal identifiers, such data are then referred as anonymous data. Some studies cannot be performed with anonymous data particularly when researcher would need to link between the two, data and the records. The public health researcher often distinguish itself from medicine based, curative research trials, rather it is concerned principally with the population and committees and their problem and sufferings in general rather than individuals and prevention and the cure of the diseases. In many cases the best interest of the community are at the odds in preference with new members of the community example in case of STD and HIV other people may need to know that what consequences they are at risk in terms of transmissible diseases, this may apply to other studies of public health. Values of Public health and Public health Researchers, public health is primarily concerned with the health and well being of the entire population rather than individuals. Public health activities also include community collaboration for health and identifications of priorities for public health actions. Ethics in public health can be defined as the identification, analysis and resolution of ethical problems arising in public health practice as well as research. Public health ethics has a broad scope including social and ethical concerns arising in the health promotion and disease prevention as well as in the public health research. Philosophically in the public health research two theories have been considered in this regard which is utilitarian approach and the communitarian approach. Communitarian perspective may favor limiting the autonomy of an individual in the public interest for the sake of greater and common good. Deontological theories give full support to the research participants for the protection of their rights even though if because of these protective measures the research shows down, on the other hand the utilitarian approach strives to maximize the beneficial consequences. Utilitarian theories strongly appreciate the public health programs and favor them with strong justifications important and compulsory programs example, vaccination programs for children and certain program for public water supply. Although in both research areas which are public health as well as clinical health this point has its own relevance and importance, but we have already discussed in detail the public health projects which are to be taken care under the umbrella of health care system with entire approach of either preventive care on the broader side but also the curative part is been catered may at times in public health research paradigm, but talking under the cover of public health means catering not only the public health related issues but also other factors that somehow directly or indirectly influence health or even other social and community based issues may come under the heading of public health. Public health programmed by enlarge are implemented in no emergency situations and therefore in case of emergency the outbreak data may be collected in each case. Public health program for example in antismoking campaign, data are collected to evaluate the efficacy and also other characteristics of the program. Increasing public health efforts are being focused in order to strengthen the communities for focusing or capacity building which involves educational and developmental projects which may facilitate the community in long term.

In order to involve the community member who have specialized knowledge, skills, and capabilities to carry out different roles in from amongst themselves and the capacity building of the community would help individuals to understand their own needs and demands in regard of their values as appropriately. Often the public health planners and policy makers are required to balance their professional responsibilities to use sound research methods and meaningful opportunities for the communities which will keep the (almost) entire population of the community active and engaged in the process. Community based practice encourages the strategic based policy and cooperation of public and private health and social sciences, agencies, local funding resources and authorities, business community, neighboring infrastructures, schools, universities and other education providing systems, public officials, and public agencies, consumer commodities, epidemiological understanding of the community's health and well being and other interventions that recognize the need and importance of health related issues for an individual and the community as a whole. 
This work is licensed under Creative Commons Attribution 4.0 Licens

DOI: 10.19080/IJCSMB.2017.02.555598
Your next submission with Juniper Publishers will reach you the below assets

- Quality Editorial service

- Swift Peer Review

- Reprints availability

- E-prints Service

- Manuscript Podcast for convenient understanding

- Global attainment for your research

- Manuscript accessibility in different formats ( Pdf, E-pub, Full Text, Audio)

- Unceasing customer service

Track the below URL for one-step submission https://juniperpublishers.com/online-submission.php 
